\title{
Parallel carbon nanotube quantum dots and their interactions
}

\author{
Karin Goß,,$^{1,}$ Martin Leijnse, ${ }^{2}$ Sebastian Smerat, ${ }^{3}$ Maarten R. Wegewijs, ${ }^{4,5}$ Claus M. Schneider, ${ }^{1}$ and Carola Meyer ${ }^{1}$ \\ ${ }^{1}$ Peter Grünberg Institut (PGI-6), Forschungszentrum Jülich \& JARA Jülich Aachen Research Alliance, 52425 Jülich, Germany \\ ${ }^{2}$ Center for Quantum Devices, Niels Bohr Institute, University of Copenhagen, Universitetsparken 5, 2100 Copenhagen, Denmark \\ ${ }^{3}$ Physics Department, Arnold Sommerfeld Center for Theoretical Physics, Ludwig-Maximilians-Universität München, \\ 80333 München, Germany \\ ${ }^{4}$ Peter Grünberg Institut (PGI-2), Forschungszentrum Jülich \& JARA Jülich Aachen Research Alliance, 52425 Jülich, Germany \\ ${ }^{5}$ Institute for Theory of Statistical Physics, RWTH Aachen, 52056 Aachen, Germany
}

(Received 29 August 2012; published 23 January 2013)

\begin{abstract}
We present quantum transport measurements of interacting parallel quantum dots formed in the strands of a carbon nanotube rope. In this molecular quantum dot system, transport is dominated by one quantum dot, while additional resonances from parallel side dots appear, which exhibit a weak gate coupling. This differential gating effect provides a tunability of the quantum dot system with only one gate electrode and provides control over the carbon nanotube strand that carries the current. By tuning the system to different states, we use quantum transport as a spectroscopic tool to investigate the interdot coupling and show a route to distinguish among various side dots. By comparing the experimental data with master-equation calculations, we identify conditions for the tunneling rates that are required in order to observe different manifestations of the interdot coupling in the transport spectra.
\end{abstract}

DOI: 10.1103/PhysRevB.87.035424

PACS number(s): 73.22.-f, 73.63.Fg, 73.23.Hk

\section{INTRODUCTION}

Carbon nanotubes (CNTs) are a versatile material for electronics. In addition to extraordinary electronic ${ }^{1-4}$ and thermal $^{5-7}$ transport properties, they are mechanically flexible and strong. ${ }^{8-10}$ The current is carried by the $\pi$ orbitals of the macromolecule and, thus, the electronic transport can be strongly influenced by the environment. This feature can be exploited by using carbon nanotubes as the functional element in detectors, e. g., gas sensors. ${ }^{11,12}$ A different way of using this property is the functionalization of individual CNTs with molecules to create new hybrid types of nanodevices such as biosensors ${ }^{13,14}$ or spin valves. ${ }^{15}$

The interactions involved in such a functionalization are not yet fully understood but are expected to also play an important role in the transport properties of other interacting $\pi$ systems such as graphene or individual molecules. Much effort has been put into studying quantized transport in these systems. ${ }^{16-29}$ In contrast to graphene and individual molecules, quantum transport on clean, individual CNTs today is well established and understood. ${ }^{30-35}$ Therefore, it can be used as a spectroscopic tool to investigate more complex multicomponent devices. Within this context, CNTs bundled together in the form of a rope represent a generic and readily available system to study the electrical transport of interacting molecules.

Recently, we investigated the electronic hybridization between the parallel quantum dots (QDs) in a CNT rope system, when two electrochemical potentials of the dots are in resonance. ${ }^{36}$ Here, we report on the same device, focusing, however, on the off-resonance case in order to create a complete picture of the manifold of effects that can arise in a system of parallel coupled quantum dots. Tuning the system from in-resonance to off-resonance cases is possible by a differential gating effect which enables one to shift the dot potentials relative to each other. This effect forms a major part of this article, since it allows one to find a generic route to distinguish several quantum dots by their coupling to the environment. Previously, we reported on a selective hybridization of in-resonance spin states in a magnetic field..$^{36}$ Here, on the other hand, we use the transport data at high magnetic field to extract relative tunnel rates and discuss their effect on the stability diagram. In addition, we present the influence of a capacitive coupling on a main resonance. The experimentally observed shift of resonances can be explained with the model developed previously. ${ }^{36}$ This confirms its potential to fully describe a system of several parallel quantum dots which exhibit a tunnel coupling as well as a capacitive coupling.

This manuscript is structured as follows. After indicating the sample fabrication and measurement techniques in Sec. II, we evaluate in Sec. III the coupling and interaction parameters of four parallel quantum dots within the CNT rope. This section also includes a discussion of the differential gating effect, which allows the system to be tuned into in-resonance and off-resonance states. Section IV compares master-equation calculations with quantum transport measurements in order to show the effect of relative tunnel rates of parallel quantum dots. At last, in Sec. V, we briefly outline how to extract the true capacitive coupling between parallel quantum dots in the presence of a strongly asymmetric coupling to one lead.

\section{SAMPLE AND METHODS}

We show quantum transport measurements of a CNT rope device with the distance between the gold contacts being patterned as $360 \mathrm{~nm}$ (see Fig. 1). The highly doped Si substrate with $\mathrm{SiO}_{2}$ on top of it acts as a backgate. The CNTs are grown on substrate by the chemical vapor deposition method using $\mathrm{Fe} / \mathrm{Mo}$ as catalyst and methane as the carbon precursor. ${ }^{37}$ The growth temperature is $920^{\circ} \mathrm{C}$, where a predominant growth of single-walled carbon nanotubes and a small fraction of doublewalled CNTs is expected. ${ }^{38}$ The height profile taken from the atomic force micrograph in Fig. 1 in the quantum dot region 


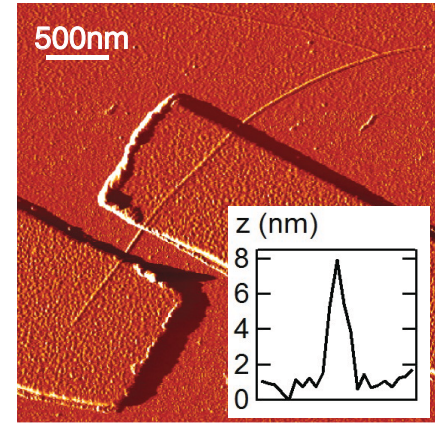

FIG. 1. (Color online) Atomic force micrograph of the parallel quantum dot device with gold contacts patterned on top of a CNT rope. Inset: Height profile $z$ in the quantum dot region between the contacts.

between the two contacts gives a height of $\sim 7 \mathrm{~nm}$. We can rule out the presence of an individual multiwalled CNT with a large diameter based on Raman scattering measurements performed on the device reported here. ${ }^{39}$ We infer from these experiments that the device consists of a bundle of several carbon nanotubes with diameters between 0.6 and $1.3 \mathrm{~nm}$ in the quantum dot region. The device exhibits a resistance of $290 \mathrm{k} \Omega$ at room temperature with a linear current-voltage characteristic indicating its metallic character. Low-temperature transport measurements are performed in a dilution refrigerator at a base temperature of $\sim 30 \mathrm{mK}$.

\section{MULTIPLE PARALLEL QUANTUM DOTS}

Figure 2 shows the stability diagram of the CNT rope device measured at low temperatures, which exhibits Coulomb diamonds as typical Coulomb blockade signatures and more atypical additional resonances. Previously, we have shown that the observable features can be explained by a formation of interacting parallel quantum dots in different strands of the rope. ${ }^{36}$ The Coulomb diamonds in Fig. 2 originate from one quantum dot which we label as the main dot and the secondary

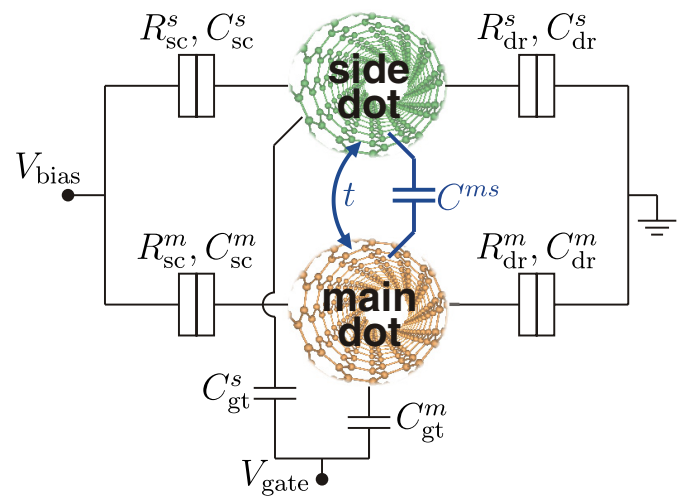

FIG. 3. (Color online) Circuit diagram of a system of two parallel quantum dots, main dot $m$ and side dot $s$, formed in two CNTs. The tunnel barriers connecting them to the same source (sc) and drain (dr) electrodes are characterized by a resistance $R$ and a capacitance $C$. The quantum dots interact via a tunnel coupling with the hybridization amplitude $t$ and a capacitive coupling with the capacitance $C^{m s}$.

resonances marked by arrows originate from side dots formed in parallel CNTs within the rope. These secondary resonances are part of a Coulomb diamond pattern with smaller slopes of the diamond edges due to a weaker backgate coupling of the side dots.

Within the gate voltage range plotted in Figs. 2(a) and 2(b), indications of three different side dots are observed as we will discuss in the following. Three main signatures are used to discriminate these three quantum dots. First, anticrossings caused by a tunnel coupling between quantum dot states on different dots can be observed at meeting points between resonance lines. This tunnel coupling is characterized by a hybridization amplitude $t$ as sketched in the circuit diagram of the quantum dot system in Fig. 3. Second, a capacitive coupling characterized by the capacitance $C^{m s}$ between the dots leads to a voltage shift $\Delta V(i)$ in the secondary resonances when proceeding from one Coulomb diamond to the next. In the stability diagram in Fig. 2(a), this voltage shift is illustrated
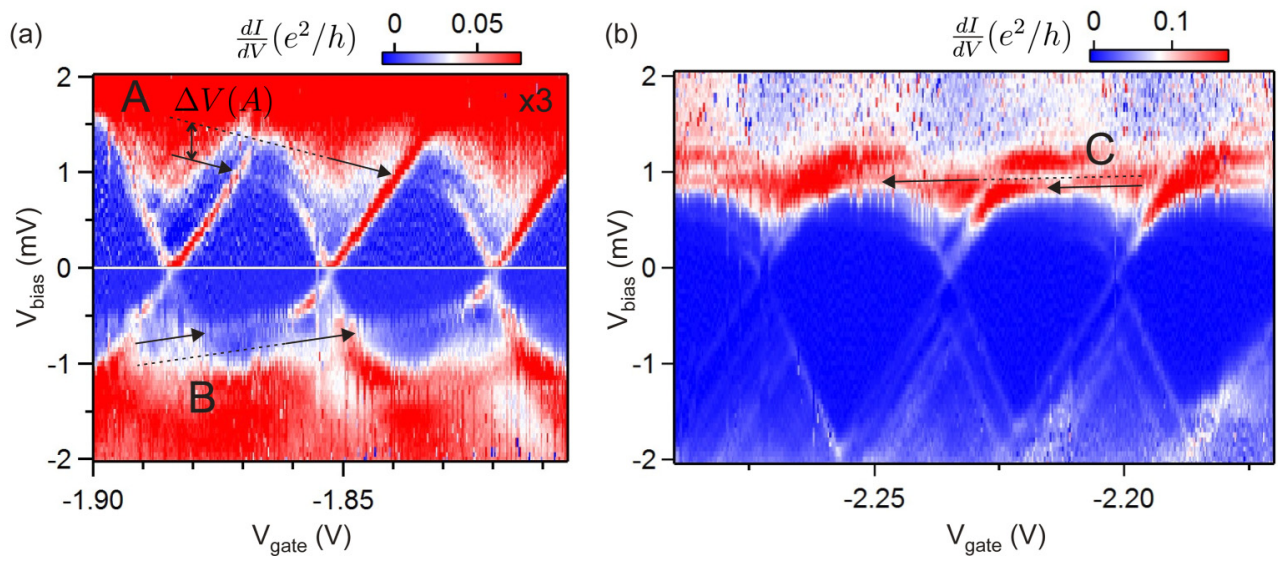

FIG. 2. (Color online) Differential conductance plot showing several charge states of the main dot. Secondary resonances of three different side dots $A, B$ (a) and $C$ (b) are indicated by arrows. The slopes of the arrows represent the slope of the respective secondary resonance, which is used for evaluating the coupling of the quantum dot to the electrodes. The resonances in subsequent main dot charge states are shifted by $\Delta V(i)$ $(i=A, B, C)$ due to a capacitive interdot coupling, which is indicated for side $\operatorname{dot} A$ as an example. Note that the differential conductance at positive bias voltage in (a) is multiplied by 3 . 
TABLE I. Coupling and interaction parameters of the quantum dots observed in Fig. 2.

\begin{tabular}{llllcc}
\hline \hline QD $i$ & $\alpha_{\mathrm{gt}}$ & $\alpha_{\mathrm{sc}}$ & $\alpha_{\mathrm{dr}}$ & $|t|(\mathrm{meV})$ & $\Delta V(i)(\mathrm{meV})$ \\
\hline Main dot & 0.05 & 0.36 & 0.59 & - & - \\
Side dot $A$ & 0.019 & 0.765 & 0.216 & 0.1 & 0.40 \\
Side dot $B$ & 0.007 & 0.074 & 0.919 & 0.075 & 0.20 \\
Side dot $C$ & 0.001 & 0.002 & 0.997 & $\neq 0$ & 0.07 \\
\hline \hline
\end{tabular}

by a vertical shift of the arrows indicative of the secondary resonances of side $\operatorname{dot} A$. The reason for such an energy shift of a side dot level is a potential change on the side dot due to addition of an electron to the main dot.

Third, the bias coupling, which is due to the interaction of the QDs with the leads, can be deduced from the slopes of the diamond edges in the stability diagram. These are represented by the slopes of the arrows indicating the secondary resonances in Fig. 2. In our experiments, the bias voltage is applied asymmetrically, i.e., the drain is kept grounded and the full bias window is applied at the source electrode. The slopes of the diamond edges of each quantum dot then are given as $+\frac{\alpha_{\mathrm{gt}}}{1-\alpha_{\mathrm{sc}}}$ and $-\frac{\alpha_{\mathrm{gt}}}{\alpha_{\mathrm{sc}}}$ for the positive and the negative slope, respectively. ${ }^{40}$ Here, $\alpha_{j}=C_{j} / C$ is the dimensionless coupling parameter of the quantum dot to electrode $j$, where $C=\sum C_{j}$ is the total capacitance as defined for a single quantum dot within the constant interaction picture. ${ }^{41}$ From this, the gate coupling is obtained as $\alpha_{\mathrm{gt}}=\frac{C_{\mathrm{gt}}}{C_{\mathrm{sc}}+C_{\mathrm{dr}}+C_{\mathrm{gt}}}$ in our case and the source coupling as $\alpha_{\mathrm{sc}}=\frac{C_{\mathrm{sc}}}{C_{\mathrm{sc}}+C_{\mathrm{dr}}+C_{\mathrm{gt}}}$, correspondingly.

The coupling parameters $\alpha_{j}$ deduced from Fig. 2 are summarized in Table I. The parameters of the main dot are directly extracted from the observed diamond pattern. For the side dots, it is not possible to observe complete Coulomb diamonds. Considering each secondary resonance separately, a large bias coupling, i.e., a strong coupling to either the source or the drain electrode, has to be considered. In the case of a large source coupling $\left(\alpha_{\mathrm{sc}} \gg \alpha_{\mathrm{dr}}\right)$, the positive slope becomes very steep, while the negative slope becomes flat. On the other hand, if the coupling to the drain electrode is large, the opposite will be observed: the positive slope is flat, while the negative slope is steep. We interpret each secondary resonance to be the flat slope of a diamond pattern, hence, belonging to different side dots, labeled as $A, B$, and $C$ in Fig. 2. The second slope of each of these parallel Coulomb diamonds cannot be smaller than the one of the main dot diamond pattern, because it would be observable as a secondary resonance with the same anticrossing gap and capacitive voltage shift. Since this is not observed in our data, we assume the second slope to be equal to or steeper than the main dot resonances. Due to its steepness, it is then obscured by the prominent main diamond pattern. In order to obtain an estimate for the coupling of the side dots to the electrodes, we assumed values for this slope between a minimum value, equal to the main diamond slopes, and a maximum value corresponding to a vertical slope. Within this range, the results for the coupling parameters do not change significantly.

The coupling parameters found by this analysis are given in Table I and assume the respective second slope of the side dot diamonds to be equal to that of the main diamond. For side $\operatorname{dot} A$ we find a strong source coupling, while side dots $B$ and $C$ exhibit a strong drain coupling. The coupling to the gate electrode is similar for side dots $B$ and $C$, while it is slightly stronger for side $\operatorname{dot} A$. A screening of particular CNTs by surrounding CNTs within the rope is a possible reason for a different coupling to the backgate. Furthermore, the asymmetric coupling to one of the contacts is a clear indication for different interface properties at the leads, which can originate from a changing assembly of the rope along the QD region. The physics of the effects that we observe in the system of coupled parallel QDs is similar to the dynamical channel blockade that has been studied for a three-terminal quantum dot. ${ }^{42}$

Although all quantum dots in the system are controlled by the same gate electrode, the dot-dependent backgate coupling creates a differential gating effect that is used to tune the system into various states. This is illustrated in Fig. 4 for one main dot (red) and one side dot (green) where interactions between the QDs are neglected for simplicity. At the first indicated position in the stability diagram in Fig. 4(a), the bias voltage and the gate voltage $V_{\text {gate }}^{1}$ are set such that tunneling via both quantum dots is possible simultaneously. The level alignment for this situation is sketched in the left panel of Fig. 4(b): While the level on the main dot is in resonance with the chemical potential of the drain (negative slope of the Coulomb diamond), the level on the side dot is in resonance with the chemical potential of the source (positive slope of the secondary resonance). Keeping the bias voltage fixed and

(a)

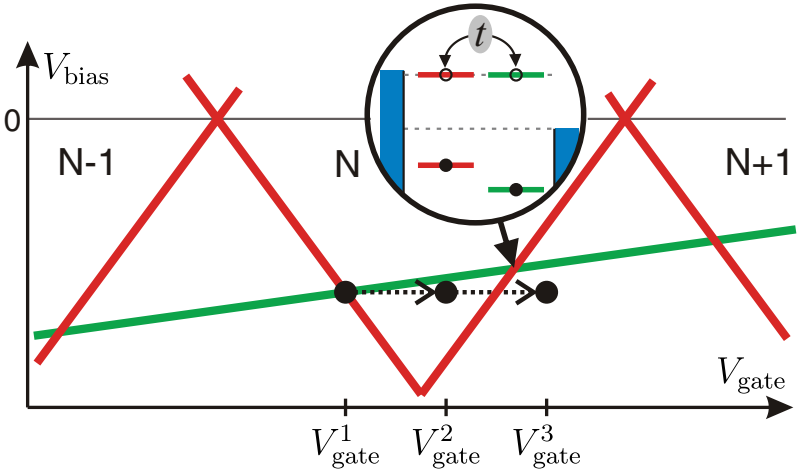

(b)

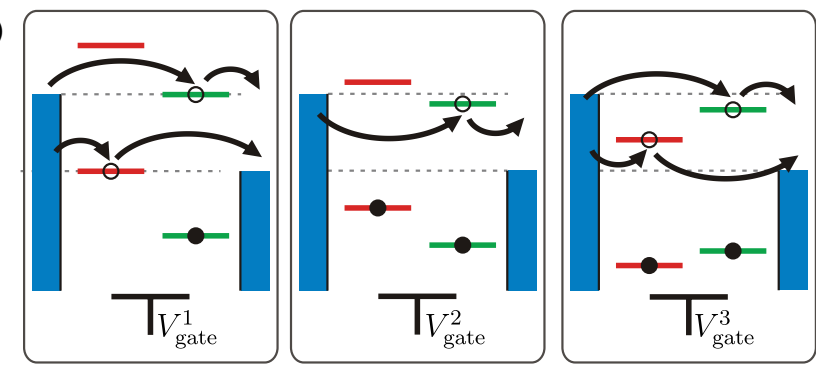

FIG. 4. (Color online) (a) Schematic stability diagram of a main dot (red) in parallel to one side dot (green) in the region of negative bias voltage. (b) Level alignment for three gate voltages as indicated in (a) at a constant bias voltage. At $V_{\text {gate }}^{1}$, tunneling via both quantum dots is possible. Due to the differential gating, it is possible to add one more electron onto the main dot, while the side dot occupancy does not change for $V_{\text {gate }}^{2}$. At $V_{\text {gate }}^{3}$, again tunneling via two dots (but different states) is possible. 
increasing the gate voltage to $V_{\text {gate }}^{2}$ lowers the main dot level below the bias window and fills the main dot permanently with one more electron [see central panel of Fig. 4(b)]. The main dot is in Coulomb blockade, but tunneling via the side dot level is still possible. This is a particular property of the parallel quantum dot system in contrast to a setup with serial quantum dots. There, the transport via the whole device is fully blockaded when only one of the dots is in Coulomb blockade.

Increasing the gate voltage further to $V_{\text {gate }}^{3}$ tunes the next higher energy level of the main dot into the bias window allowing again tunneling via both quantum dots in parallel. While the level on the main dot had a higher energy than the side dot level at $V_{\text {gate }}^{2}$, it exhibits a lower energy at $V_{\text {gate }}^{3}$, which (with only one gate electrode) is solely possible with a differential gating effect. The situation where the electronic states of the two quantum dots are at the same energy, i.e., in resonance with each other, is depicted in the inset of Fig. 4(a). To probe this in-resonance state and a possible hybridization of quantum dot levels, both levels need to be simultaneously in resonance with one of the electrode chemical potentials. From earlier investigations we know that the current is carried by the bonding state of hybridized levels and that the wave-function overlap is symmetric. ${ }^{36}$

Hence, the differential gating effect as it is observed here, can be used to tune the quantum dot system into in-resonance and off-resonance states and provides a control over which strand of the rope carries the current. This enables us to use quantum transport as a spectroscopic tool for probing various properties of the system.

Now that it is clear how the differential gating effect can be employed to characterize the parallel quantum dot system, we want to use it to distinguish between the side dots by evaluating their interaction parameters in the following. From the discrete shift of a secondary resonance $\Delta V(i)$ between two subsequent main dot Coulomb diamonds, the capacitive interdot coupling can be extracted. This shift is found to be twice as large for resonance $A$ in comparison to resonance $B$ (see Table I). For side $\operatorname{dot} C$, an even smaller capacitive coupling is found. The second interaction is the tunnel coupling to the main dot, as mentioned above. A hybridization between states on two different quantum dots causes an anticrossing between resonance lines. The gap between a bonding and an antibonding state observable at the anticrossings corresponds to $\Delta E=2|t|$, where $t$ is the hybridization amplitude. ${ }^{36}$ From a high-resolution measurement (shown in Ref. 36), $|t|$ can be estimated to be 0.1 and $0.075 \mathrm{meV}$ for $A$ and $B$, respectively. For side $\operatorname{dot} C$, a magnitude for the hybridization amplitude cannot be accurately determined. However, the clear bending of resonance lines indicate that a hybridization indeed occurs also for these quantum dot states. Hence, the different magnitude of the interactions between the quantum dots gives evidence that Fig. 2 contains the fingerprints of one main dot connected in parallel to three side dots.

A further evidence for interpreting the secondary resonances as three side dots can be given from a distinct evolution with increasing magnetic field. Figures 5(a) and 5(b) show the stability diagram of the main dot and the secondary resonance $B$ at a magnetic field $B=0 \mathrm{~T}$ and $2 \mathrm{~T}$, respectively. While at $B=0 \mathrm{~T}$ the secondary resonance is passing the Coulomb blockaded region at $V_{\text {bias }} \approx-0.6 \mathrm{mV}$, it appears at a more negative bias voltage for $B=2 \mathrm{~T}$. Figure 5(c) presents several charge states of the main dot at $B=10 \mathrm{~T}$. At this high magnetic field, no secondary resonances can be observed at negative bias voltages, where the signatures of side dot $B$ could be measured at zero magnetic field (see Fig. 2). In contrast, enhanced secondary resonances can be observed at positive (a)

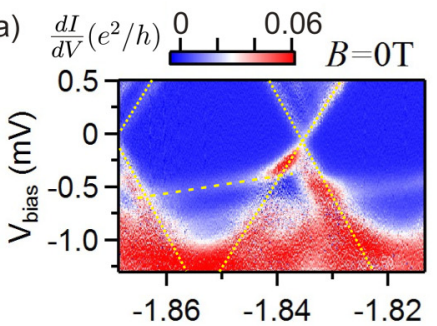

(b)

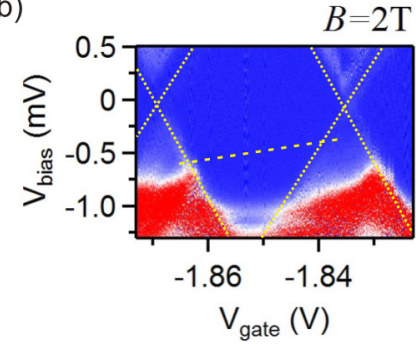

(c)

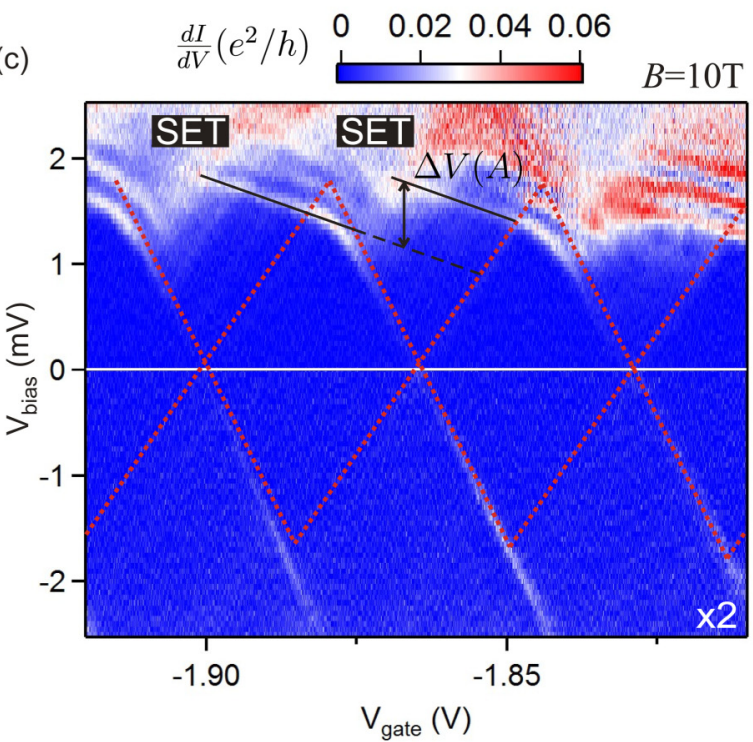

FIG. 5. (Color online) Differential conductance plots for magnetic field (a) $B=0 \mathrm{~T}$, (b) $B=2 \mathrm{~T}$, and (c) $B=10 \mathrm{~T}$. (b) The main and secondary resonances as observed at $B=0 \mathrm{~T}$ are indicated in yellow. (c) Note that the differential conductance at negative bias voltage is multiplied by 2 . For better visibility, main dot Coulomb diamonds are indicated in red. Secondary resonances of side dot $A$ indicated by black lines with the same slope are visible in the single electron tunneling (SET) region of the main dot. The shift $\Delta V(A)$ of one resonance is indicated by the arrow. 
bias voltage, indicating electrons tunneling favorably via side $\operatorname{dot} A$. The contrary evolution of the secondary resonances in a magnetic field confirms once again the presence of several parallel side dots.

Comparing the secondary resonances $A$ in Fig. 5(c) with those in Fig. 2(a), two differences can be observed in the single electron tunneling (SET) region of the main dot. First, excited states of side $\operatorname{dot} A$ are visible, which cause parallel secondary resonances with equal coupling and interaction parameters. A detailed discussion concerning these excited states can be found elsewhere. ${ }^{36}$ Second, the voltage shift $\Delta V(A)$ of the secondary resonance is found to occur within the SET regime [see the arrow in Fig. 5(c)] and not at the edge of the subsequent Coulomb diamond. This effect is best visible for the secondary resonance with the lowest energy and has its origin in the relative tunneling rates of the main dot and the side dots, which will be discussed in the next section.

In this first section, we showed how a differential gating effect enables the identification and characterization of several parallel quantum dots. A strong bias coupling is found for the side dots in the setup. By tuning to in-resonance and offresonance states of the system, various interactions can be probed. Furthermore, the differential gating is used to control which one of the strands within the rope carries the current.

\section{RATE-DEPENDENT QUANTUM TRANSPORT FEATURES}

Figure 6 depicts two possible observations in the SET region of a main dot in parallel to a capacitively coupled side dot (a hybridization of quantum dot states is neglected for simplicity). In the first case (a), the voltage shift $\Delta V(i)$ occurs exactly at the diamond edge of the right diamond, where an additional electron is filled onto the main dot. The second case (b) shows the up-shifted secondary resonance already in the SET regime of the main dot. This observation can be made if an electron resides for a long time on the main dot state within the bias window due to a reduced tunneling rate towards the drain electrode. This is the effect mentioned in Sec. III which can be observed in Fig. 5(c) for the main dot and side dot $A$. In a magnetic field $(B=10 \mathrm{~T})$, the conductance via the main
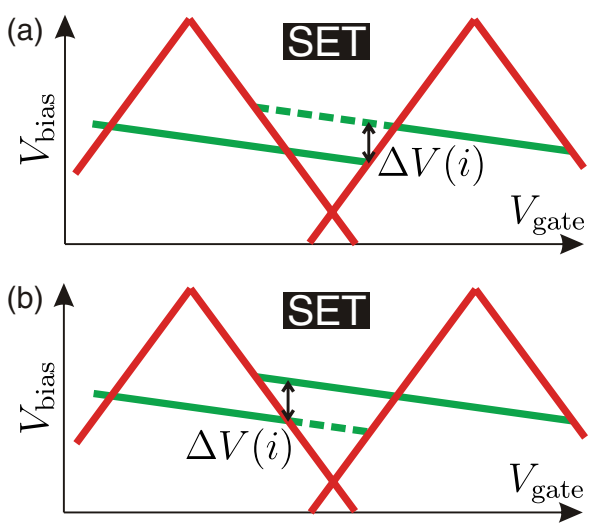

FIG. 6. (Color online) Schematic stability diagram of a main dot (red) in parallel to one side dot (green) in the region of positive bias voltage. The voltage shift $\Delta V(i)$ occurs in (a) at the Coulomb diamond edge and in (b) in the SET regime of the main dot.

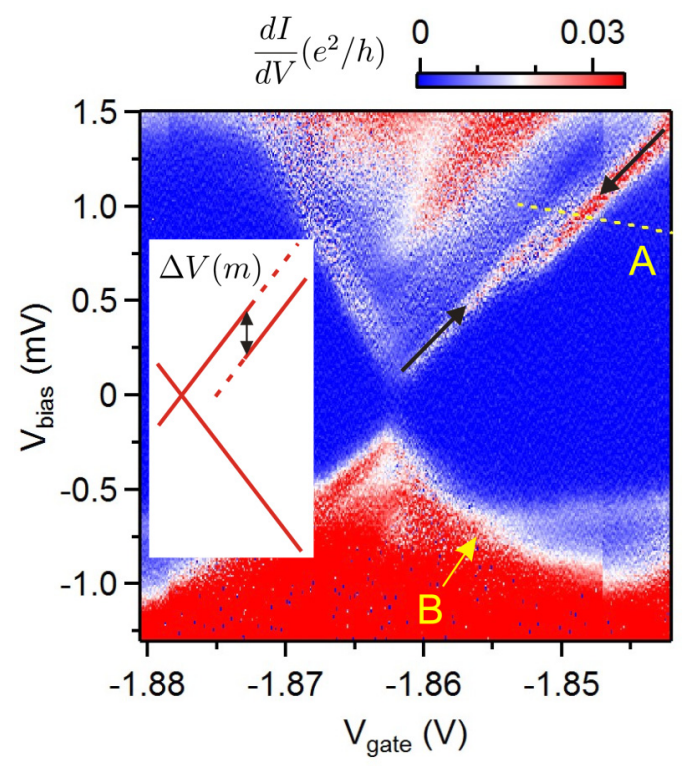

FIG. 7. (Color online) Differential conductance plot exhibiting a main resonance shifted by $\Delta V(m)$ (see black arrows and inset). The secondary resonances of side $\operatorname{dot} A$ and $B$ are indicated in yellow.

dot is very low (faint Coulomb diamonds), indicating reduced tunneling rates of the main dot. Furthermore, the shift of the secondary resonance is found to occur in the SET regime and not at the diamond edge. At lower bias voltages, where an unshifted resonance is expected (dotted line), no conductance can be measured, in analogy to Fig. 6(b).

A similar effect with the same physical origin can be observed in Fig. 7, which shows another transport measurement at $B=0 \mathrm{~T}$ at similar charge states as in Fig. 2. The secondary resonance of side $\operatorname{dot} B$ is observed similarly to the previous measurement. The position of the secondary resonance from side $\operatorname{dot} A$ is difficult to recognize due to a low conductance in this particular measurement. Instead, a shift of the diamond edge $\Delta V(m)$ can be observed close to the meeting point of the secondary resonance with the main resonance. This is, like the voltage shift in the secondary resonances, a manifestation of the capacitive coupling between the main dot and side $\operatorname{dot} A$. In a system of capacitively coupled quantum dots, the chemical potential of the quantum dots will mutually depend on each other. Hence, resonance lines of any dot-not only a side dot-are expected to shift when the number of electrons on the parallel quantum dot is changed. This means that also a situation inverse to what we have discussed so far can appear in the stability diagram. Such a situation involves the tunneling of an additional electron via a side dot state, which changes the potential for the electrons on the main dot. Then, a shifted main dot diamond edge is expected at energies higher than the respective secondary resonance, i.e., in the single electron tunneling regime of the side dot. This effect causes the observed shift in Fig. 7. As we will see in the following, the relative tunnel rates of quantum dot states are the decisive parameter for a shift of resonances.

We use master-equation calculations with the model developed in Ref. 36, describing a parallel double quantum dot (containing the main dot and side $\operatorname{dot} A$ ) within a constant interaction model ${ }^{43}$ extended to account for interactions 


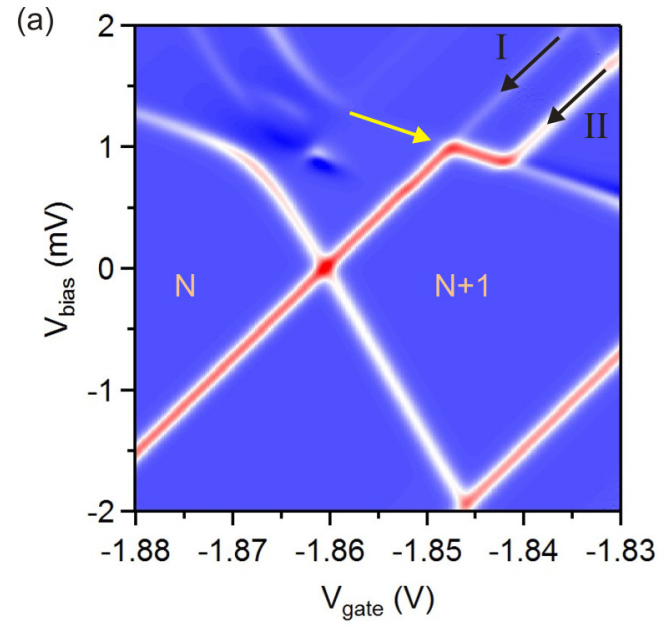

(b)

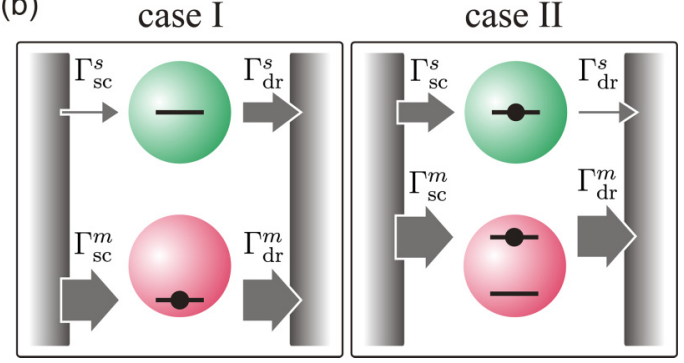

FIG. 8. (Color online) (a) Differential conductance calculated by master equations using the model of two interacting parallel quantum dots. Black arrows indicate the shift of the main resonance appearing at the secondary resonance (yellow arrow). (b) Schematic drawing of electron tunneling via two parallel CNT quantum dots. $\Gamma_{\mathrm{sc} / \mathrm{dr}}^{s}$ and $\Gamma_{\mathrm{sc} / \mathrm{dr}}^{m}$ are the tunneling rates from source (sc) and drain (dr) to the side dot $s$ (green) and the main dot $m$ (red), respectively. (I) If $\Gamma_{\mathrm{sc}}^{s} \ll \Gamma_{\mathrm{dr}}^{s}$, the side dot is mainly unoccupied and electrons can tunnel via the main dot like in an uncoupled quantum dot. (II) In the case of $\Gamma_{\mathrm{sc}}^{s} \gg \Gamma_{\mathrm{dr}}^{s}$, an additional electron resides on the side dot most of the time and electrons tunneling via the main dot can only tunnel via a state shifted in energy by the capacitive interdot coupling.

between the quantum dots. Figure 8(a) shows a calculated stability diagram according to this model. Similarly to the measurement, the main dot diamond edge is shifted at the crossing of the secondary resonance. The calculations assume a larger capacitive coupling than observed in the experiment in order to enhance the visibility of the effect.

In the calculated stability diagram, the shifted main dot diamond edge appears only for particular relative tunneling rates from the leads to the quantum dots. The following discussion assumes one state on each quantum dot to be within the bias window and the applied bias voltage to be positive. The left panel (case I) of Fig. 8(b) depicts a situation where the tunneling rates for the state on the side dot are $\Gamma_{\mathrm{sc}}^{s}<\Gamma_{\mathrm{dr}}^{s}$, while $\Gamma_{\mathrm{sc}}^{m}=\Gamma_{\mathrm{dr}}^{m}>\Gamma_{\mathrm{dr}}^{s}$ is assumed for the main dot. The side dot state then is mainly unoccupied, because electrons will immediately tunnel out of the dot into the drain. Therefore, electrons tunneling via the main dot will not experience an additional potential, because the probability to have an electron on the side dot at the same time is low. As a result, the diamond edge in the stability diagram will appear as a continuous resonance line and exhibit no shift as for uncoupled QDs [see resonance I in Fig. 8(a)].

For the calculation shown in Fig. 8(a), the tunneling rates for the side dot are assumed to be reversed, i.e., $\Gamma_{\mathrm{sc}}^{s}>\Gamma_{\mathrm{dr}}^{s}$, as depicted in the right panel (case II) of Fig. 8(b). For the main dot, $\Gamma_{\mathrm{sc}}^{m}=\Gamma_{\mathrm{dr}}^{m}>\Gamma_{\mathrm{sc}}^{s}$ is assumed. This leads to the following situation in the SET regime (i.e., above the side dot resonance): The state on the side dot is mainly occupied, and transport predominantly takes place via a state on the main dot, which is shifted in energy due to the interdot capacitive coupling. The conductance in the original diamond edge (side dot not occupied) then is suppressed, whereas the shifted diamond edge (side dot occupied) appears enhanced, which causes the higher conductance in resonance II in Fig. 8(a). This configuration of tunneling rates also suppresses the conductance through the side dot; hence, the secondary resonance appears less pronounced. This is consistent with the experimentally found weak conductance of the secondary resonance of side $\operatorname{dot} A$ in Fig. 7.

In conclusion, by reproducing the experimental observations with master-equation calculations, we have shown that the energy and the conductance of the resonances in the stability diagram of interacting parallel quantum dots strongly depend on the tunnel rates into particular quantum dot states. Hence, the tunneling rates of one quantum dot dictate the relative conductance for the resonances of the parallel quantum dot and are decisive for the observation of their energy splitting.

\section{EVALUATING THE TRUE CAPACITIVE COUPLING STRENGTH}

As we have shown above, a shift in the resonance lines of the side dot as well as of the main dot is a measure of the capacitive coupling between parallel quantum dots. However, here we find the magnitude of the shift of the main resonance to be $\Delta V(m) \approx 0.2 \mathrm{meV}$, whereas $\Delta V(A) \approx 0.4 \mathrm{meV}$ is obtained from the voltage shift of the secondary resonance. This discrepancy can be explained by considering the difference in the bias coupling of side $\operatorname{dot} A$ and the main dot. In fact, the voltage offset due to a capacitive interdot coupling and also the magnitude of the anticrossing gap depend on the bias coupling, which we described in Sec. III. A large $\alpha_{\mathrm{sc}}$ will increase the observable shift of resonances, because $\Delta V(i)=U^{m s} /\left[1-\alpha_{\mathrm{sc}}(i)\right]$, where $\alpha_{\mathrm{sc}}$ is the source coupling of the dot corresponding to the shifting resonance and $U^{m s}$ is the true capacitive coupling strength. The values of the shifts are, thus, always upper bounds for the actual capacitive interdot coupling.

Considering the source coupling of the main dot and the side $\operatorname{dot} A$ in Table I, a shift of

$$
\Delta V(m)=\Delta V(A) \frac{1-\alpha_{\mathrm{sc}}(A)}{1-\alpha_{\mathrm{sc}}(m)}=0.15 \mathrm{meV}
$$

is expected for the main dot resonance. This value is comparable to the $\Delta V(m)$ deduced from the shift of the main resonance, taking into account the experimental error. In principle, the source coupling of the main dot is more reliable than the ones of the side dots, because no assumptions had to be made for obtaining them. Hence, we evaluate the true capacitive interdot 
coupling between main dot and side $\operatorname{dot} A$ from the shifted main diamond edge and obtain $U^{m A}=0.13 \mathrm{meV}$. Although several side dots are interacting with the main dot, only side dot $A$ is coupled strongly enough to shift the main dot resonances in energy.

\section{SUMMARY AND CONCLUSIONS}

In summary, we discussed a multicomponent molecular transport device employing quantum transport as a spectroscopic tool. We use a carbon nanotube rope as a molecular model system, which exhibits rich characteristics in its transport spectra. The formation of multiple parallel quantum dots is concluded from secondary resonances. A differential gating effect due to a dot-dependent gate coupling allows for tuning the quantum dot system into various states using only one gate electrode. By tuning to in- and off-resonance states of the system, quantum transport spectroscopy can distinguish several parallel quantum dots by their coupling and interaction properties, as we have shown on three side dots coupled in parallel to one main dot. Furthermore, the differential gating provides a control over the carbon nanotube strand carrying the current.

The distinct coupling of the quantum dots to the leads, their interactions, and the impact of relative tunneling rates lead to a variety of features in the transport spectra which we discussed in depth. By reproducing the experimental data with master-equation calculations, we have identified the requirements for observing an energy offset due to a possible interaction between parallel quantum dots. Concentrating on the off-resonance quantum transport, we have found that a capacitive interdot coupling is not only observable in a shift of secondary resonances but, additionally, can manifest itself in shifting the edges of otherwise regular Coulomb diamonds. The exact position and appearance of such a shift depends on the relative tunneling rates through the parallel quantum dots.

Our results provide the basis for understanding quantum transport via parallel quantum dots, which is an important issue in molecular transport where a large variety of hybrid transport devices with new functionalities are expected.

\section{ACKNOWLEDGMENTS}

We thank H. Schoeller and U. Schollwöck for fruitful discussions and S. Trellenkamp for e-beam writing, as well as S. Estévez Hernández, H. Kertz, H. Pfeifer, W. Harneit, and J. Lauer for technical assistance. We acknowledge the DFG (FOR 912) and the European Union Seventh Framework Programme (FP7/2007-2013) under Agreement No. 270369 (ELFOS) for funding.
*Current address: 1. Physikalisches Institut, Universität Stuttgart, Pfaffenwaldring 57, 70550 Stuttgart, Germany; karin.goss@pi1.physik.uni-stuttgart.de

${ }^{1}$ C. T. White and T. N. Todorov, Nature 393, 240 (1998).

${ }^{2}$ J. W. G. Wildöer, L. C. Venema, A. G. Rinzler, R. E. Smalley, and C. Dekker, Nature 391, 59 (1998).

${ }^{3}$ J. Kong, E. Yenilmez, T. W. Tombler, W. Kim, H. Dai, R. B. Laughlin, L. Liu, C. S. Jayanthi, and S. Y. Wu, Phys. Rev. Lett. 87, 106801 (2001).

${ }^{4}$ W. Liang, M. Bockrath, D. Bozovic, J. H. Hafner, M. Tinkham, and H. Park, Nature 411, 665 (2001).

${ }^{5}$ P. Kim, L. Shi, A. Majumdar, and P. L. McEuen, Phys. Rev. Lett. 87, 215502 (2001).

${ }^{6}$ M. Fujii, X. Zhang, H. Xie, H. Ago, K. Takahashi, T. Ikuta, H. Abe, and T. Shimizu, Phys. Rev. Lett. 95, 065502 (2005).

${ }^{7}$ A. A. Balandin, Nat. Mater. 10, 569 (2011).

${ }^{8}$ H. J. Dai, J. H. Hafner, A. G. Rinzler, D. T. Colbert, and R. E. Smalley, Nature 384, 147 (1996).

${ }^{9}$ D. A. Walters, L. M. Ericson, M. J. Casavant, J. Liu, D. T. Colbert, K. A. Smith, and R. E. Smalley, Appl. Phys. Lett. 74, 3803 (1999).

${ }^{10}$ M. F. Yu, O. Lourie, M. J. Dyer, K. Moloni, T. F. Kelly, and R. S. Ruoff, Science 287, 637 (2000).

${ }^{11}$ J. Kong, N. R. Franklin, C. W. Zhou, M. G. Chapline, S. Peng, K. J. Cho, and H. J. Dai, Science 287, 622 (2000).

${ }^{12}$ T. Kawano, H. C. Chiamori, M. Suter, Q. Zhou, B. D. Sosnowchik, and L. Lin, Nano Lett. 7, 3686 (2007).

${ }^{13}$ K. Besteman, J.-O. Lee, F. G. M. Wiertz, H. A. Heering, and C. Dekker, Nano Lett. 3, 727 (2003).
${ }^{14}$ Y.-B. Zhang, M. Kanungo, A. J. Ho, P. Freimuth, D. van der Lelie, M. Chen, S. M. Khamis, S. S. Datta, A. T. C. Johnson, J. A. Misewich, and S. S. Wong, Nano Lett. 7, 3086 (2007).

${ }^{15}$ M. Urdampilleta, S. Klyatskaya, J.-P. Cleuziou, M. Ruben, and W. Wernsdorfer, Nat. Mater. 10, 502 (2011).

${ }^{16}$ H. Park, J. Park, A. K. L. Lim, E. H. Anderson, A. P. Alivisatos, and P. L. McEuen, Nature 407, 57 (2000).

${ }^{17}$ J. Park, A. N. Pasupathy, J. I. Goldsmith, C. Chang, Y. Yaish, J. R. Petta, M. Rinkoski, J. P. Sethna, H. D. Abruna, P. L. McEuen, and D. C. Ralph, Nature 417, 722 (2002).

${ }^{18}$ W. Liang, M. P. Shores, M. Bockrath, J. R. Long, and H. Park, Nature 417, 725 (2002).

${ }^{19}$ S. Kubatkin, A. Danilov, M. Hjort, J. Cornil, J.-L. Brédas, N. StuhrHansen, P. Hedegård, and T. Bjørnholm, Nature 425, 698 (2003).

${ }^{20}$ L. A. Ponomarenko, F. Schedin, M. I. Katsnelson, R. Yang, E. W. Hill, K. S. Novoselov, and A. K. Geim, Science 320, 356 (2008).

${ }^{21}$ F. Molitor, S. Droscher, J. Guttinger, A. Jacobsen, C. Stampfer, T. Ihn, and K. Ensslin, Appl. Phys. Lett. 94, 222107 (2009).

${ }^{22}$ A. R. Champagne, A. N. Pasupathy, and D. C. Ralph, Nano Lett. 5, 305 (2005).

${ }^{23}$ A. N. Pasupathy, J. Park, C. Chang, A. V. Soldatov, S. Lebedkin, R. C. Bialczak, J. E. Grose, L. A. K. Donev, J. P. Sethna, D. C. Ralph, and P. L. McEuen, Nano Lett. 5, 203 (2005).

${ }^{24}$ H. B. Heersche, Z. de Groot, J. A. Folk, H. S. J. van der Zant, C. Romeike, M. R. Wegewijs, L. Zobbi, D. Barreca, E. Tondello, and A. Cornia, Phys. Rev. Lett. 96, 206801 (2006).

${ }^{25}$ M.-H. Jo, J. E. Grose, K. Baheti, M. M. Deshmukh, J. J. Sokol, E. M. Rumberger, D. N. Hendrickson, J. R. Long, H. Park, and D. C. Ralph, Nano Lett. 6, 2014 (2006). 
${ }^{26}$ E. A. Osorio, K. O’Neill, M. R. Wegewijs, N. Stuhr-Hansen, J. Paaske, T. Bjørnholm, and H. S. J. van der Zant, Nano Lett. 7, 3336 (2007).

${ }^{27}$ B. Trauzettel, D. V. Bulaev, D. Loss, and G. Burkard, Nat. Phys. 3, 192 (2007).

${ }^{28}$ F. Sols, F. Guinea, and A. H. Castro Neto, Phys. Rev. Lett. 99, 166803 (2007).

${ }^{29}$ C. Stampfer, E. Schurtenberger, F. Molitor, J. Guettinger, T. Ihn, and K. Ensslin, Nano Lett. 8, 2378 (2008).

${ }^{30}$ S. J. Tans, M. H. Devoret, H. J. Dai, A. Thess, R. E. Smalley, L. J. Geerligs, and C. Dekker, Nature 386, 474 (1997).

${ }^{31}$ J. Nygård, D. H. Cobden, and P. E. Lindelof, Nature 408, 342 (2000).

${ }^{32}$ D. H. Cobden and J. Nygård, Phys. Rev. Lett. 89, 046803 (2002).

${ }^{33}$ S. Sapmaz, P. Jarillo-Herrero, J. Kong, C. Dekker, L. P. Kouwenhoven, and H. S. J. van der Zant, Phys. Rev. B 71, 153402 (2005).

${ }^{34}$ F. Kuemmeth, S. Ilani, D. C. Ralph, and P. L. McEuen, Nature 452, 448 (2008).
${ }^{35}$ G. A. Steele, G. Gotz, and L. P. Kouwenhoven, Nat. Nanotechnol. 4, 363 (2009).

${ }^{36}$ K. Goß, S. Smerat, M. Leijnse, M. R. Wegewijs, C. M. Schneider, and C. Meyer, Phys. Rev. B 83, 201403 (2011).

${ }^{37}$ J. Kong, H. T. Soh, A. M. Cassell, C. F. Quate, and H. J. Dai, Nature 395, 878 (1998).

${ }^{38}$ C. Spudat, C. Meyer, K. Goss, and C. M. Schneider, Phys. Status Solidi B 246, 2498 (2009).

${ }^{39}$ K. Goß, N. Peica, C. Thomsen, J. Maultzsch, C. M. Schneider, and C. Meyer, Phys. Status Solidi B 248, 2577 (2011).

${ }^{40}$ R. Hanson, L. P. Kouwenhoven, J. R. Petta, S. Tarucha, and L. M. K. Vandersypen, Rev. Mod. Phys. 79, 1217 (2007).

${ }^{41}$ L. P. Kouwenhoven, D. G. Austing, and S. Tarucha, Rep. Prog. Phys. 64, 701 (2001).

${ }^{42}$ A. Cottet, W. Belzig, and C. Bruder, Phys. Rev. B 70, 115315 (2004).

${ }^{43}$ Y. Oreg, K. Byczuk, and B. I. Halperin, Phys. Rev. Lett. 85, 365 (2000). 